\title{
RESTRICTED HOMOLOGICAL PROPERTIES OF MODULES
}

DAVID A. HILL

(Received 20 May 1971; revised 13 March 1972)

Communicated by G. E. Wall

\section{Introduction}

Let $R$ be a ring and $M$ a left $R$-module. We investigate when the functors $\operatorname{Hom}_{R}(M,-), \operatorname{Hom}_{R}(-, M)$, and $-\otimes_{R} M$ are exact for certain restricted subcategories of modules.

In particular, a module $N$ is defined to be injective relative to $M$ in case for every $R$ submodule $K$ of $M \operatorname{Hom}_{R}(M, N) \rightarrow \operatorname{Hom}_{R}(K, N) \rightarrow 0$ is exact. If $N=M$ in the above sequence, $M$ is said to be quasi-injective.

Dually, a module $N$ is said to be projective relative to $M$ in case for each factor module $M / K$ of $M, \operatorname{Hom}_{R}(N, M) \rightarrow \operatorname{Hom}_{R}(N, M / K) \rightarrow 0$ is exact. If $N=M$ in the above sequence, $M$ is said to be quasi-projective.

Throughout we will let $E(M)$ denote the injective hull of $M$, and $P(M)$ the projective cover of $M$, when $M$ has a projective cover. We will write $K \leqq M$ to mean that $K$ is an $R$-submodule of $M$. The Socle of $M$, which is the sum of all simple submodules of $M$, will be denoted by $\operatorname{Soc}(M)$. For any $X \leqq M$ and $A \leqq R$, the notation

$$
\begin{aligned}
& l_{R}(X)=\{a \in R \mid a \cdot X=0\} \\
& r_{M}(A)=\{m \in M \mid A \cdot m=0\}
\end{aligned}
$$

will be used to denote the left and right annihilators of $X$ and $A$ respectively. The Jacobson radical of $R$ will always be denoted by $J$.

Also we will say that a module is $\Sigma-x(\Pi-x)$ in case every direct sum (direct product) of copies of $M$ has property $x$.

In $\S 1$, we further investigate the structure of quasi-injective modules. In particular, we find some necessary conditions on a ring $R$ in order that every quasi-injective left $R$-module be $\Pi$-quasi-injective.

This work comprises a portion of a Ph. D. thesis written under the supervision of Professor F. W. Anderson and submitted to the graduate faculty of the University of Oregon in the summer of 1970. The author wishes to express his gratitude to Professor Anderson for his encouragement and many helpful suggestions. 
In $\S 2$, we study the structure of quasi-projective modules. We show that if every quasi-projective is projective modulo the annihilator then $R$ is semi-local. Also if $R$ is left perfect, we show that a left $R$-module $M$ is projective if and only if $\operatorname{Ext}_{R}^{I}(M, K)=0$ for all $K \leqq R$ where $K$ is a superfluous left ideal.

In $\S 3$ and $\S 4$, we introduce the concept of a quasi-flat module. These modules, which we characterize as being flat modulo their annihilator, are defined in terms of a duality property generalizing Lambek's characterization [25] of flatness in terms of injectivity. Also, we characterize those rings where every quasi-flat module is quasi-projective as the left perfect rings.

Throughout $R$ will denote a ring with identity. Unless otherwise stated, all modules will be left $R$-modules, and all homomorphisms will be $R$-homomorphisms.

\section{Quasi-injective modules}

We now characterize those modules which are $\Sigma$-quasi-injective. Henceforth, we use the notation $M^{(A)}$ to denote $\oplus \Sigma_{A} M$, and $M^{A}$ to denote $\Pi_{A} M$.

THEOREM 1.1. Let $M$ and $N$ be left $R$-modules and let $A$ be any set, then $M^{(A)}$ is injective relative to $N^{(A)}$ if and only if $M^{(A)}$ is injective relative to $N$.

Proof. $(\Rightarrow)$ Since $N \leqq N^{(A)}$, [27, Proposition 1] implies that $M^{(A)}$ is injective relative to $N$.

$(\Leftrightarrow)$ We will show that for $\sigma \in \operatorname{Hom}_{R}\left(E\left(N^{(A)}\right), E\left(M^{(A)}\right)\right)$ we have $\sigma\left(N^{(A)}\right)$ $\leqq M^{(A)}$. By [24, Proposition 10, p. 92], we have that for each $\alpha \in A, N_{\alpha} \leqq E\left(N_{\alpha}\right)$ $\leqq E\left(N^{(A)}\right)$ where $N_{\alpha} \simeq N$.

For $n_{\alpha} \in N$, define $\bar{n}_{\alpha} \in N^{(A)}$ via $\pi_{\beta}\left(\bar{n}_{\alpha}\right)=\delta_{\alpha_{\beta}} n_{\alpha}$. By hypothesis, [27, Proposition 1], and the obvious analogue of [20, Theorem 1.1],

$$
\sigma\left(\bar{n}_{\alpha}\right)=\left.\sigma\right|_{E\left(N_{r}\right)}\left(\bar{n}_{\alpha}\right) \in M^{(A)} .
$$

Since any $x \in N^{(A)}$ is of the form $x=\sum_{\alpha \in A} \bar{n}_{\alpha}$ where $\bar{n}_{\alpha} \neq 0$ for at most a finite number of $\alpha$ 's, we have $\sigma(x)=\sum \sigma\left(\bar{n}_{\alpha}\right) \in M^{(A)}$. Hence, it follows easily that $M^{(A)}$ is injective relative to $N^{(A)}$.

COROLlary 1.2. The left $R$-module $M$ is $\Sigma$-quasi-injective if and only if $M^{(A)}$ is injective relative to $M$ for all sets $A$.

Corollary 1.3. Let $M$ be a noetherian module. If $M$ is quasi-injective, then $M$ is $\Sigma$-quasi-injective.

Proof. By (1.1) it suffices to show that $M^{(A)}$ is $M$ injective. Consider

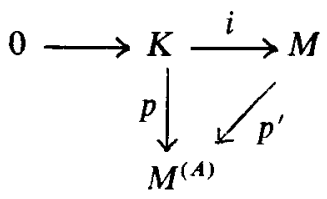


with exact row. Since $K$ is finitely generated, $p(K)$ is finitely generated. So $p(K) \leqq M^{(n)} \leqq M^{(A)}$ where $n$ is a positive integer. By [27, proposition 1] there is a map $p^{\prime}: M \rightarrow M^{(n)}$ extending $p$. Since $M^{(n)} \leqq M^{(A)}, p^{\prime}: M \rightarrow M^{(A)}$. So by (1.1) $M$ is $\sum$-quasi-injective.

It is known that left noetherian rings are precisely those rings for which every quasi-injective left $R$-module is $\Sigma$-quasi-injective. (See [10].) The following theorem improves this result.

THEOREM 1.4. A ring $R$ is left noetherian if and only if for every pair $M, N$ of left $R$-modules and every set $A, M$ is injective relative to $N$ implies $M^{(A)}$ is injective relative to $N^{(A)}$.

Proof. $(\Leftrightarrow)$ if $M$ is quasi-injective then $M^{(A)}$ is quasi-injective. So by [10, Theorem 2.3] $R$ is left noetherian.

$\Leftrightarrow$ Suppose $M$ is injective relative to $N$, and that $R$ is left noetherian. Due to (1.1), it suffices to show that $M^{(A)}$ is injective relative to $N$ for all sets $A$. Since $R$ is left noetherian, $E\left(M^{(A)}\right)=(E(M))^{(A)}$. So, it will suffice to show that if

$$
\sigma \in \operatorname{Hom}_{R}\left(E(N),(E(M))^{(A)}\right)
$$

then

$$
\sigma(N) \leqq M^{(A)} .
$$

For this it will suffice to show that for each $\alpha \in A$

$$
\pi_{\alpha} \sigma(N) \leqq M
$$

where $\pi_{\alpha}$ is the natural projection

$$
(E(M))^{(A)} \stackrel{\pi_{\alpha}}{\longrightarrow} E(M) .
$$

But $\pi_{\alpha} \sigma \in \operatorname{Hom}_{R}(E(N), E(M))$, so by the obvious analogue to [20, Theorem 1.1], $\pi_{\alpha} \sigma(n) \leqq M$, as desired.

It is known that for left artinian rings every quasi-injective is $\Pi$-quasi-injective. [See 10]. We consider the problem of characterizing those rings for which every quasi-injective module is $\Pi$-quasi-injective.

THEOREM 1.5. Let $R$ be a ring. Suppose every quasi-injective left $R$-module is $\Pi$-quasi-injective. Then $R / J$ has the following properties:

(a) Every semi-simple left $R$ module is injective as a module over $R / J$.

(b) $R / J$ is left noetherian.

Proof. It is clear that every semi-simple left $R$-module is semi-simple as an $R / J$ module. Since $R / J$ is semi-primitive, there exists a monomorphism

$$
\rho: R / J \rightarrow \prod_{a \in A} R_{\alpha}
$$


such that

$$
\pi_{\alpha} \rho(R / J)=R_{\alpha} \quad(\alpha \in A)
$$

where each $R_{\alpha}$ is a primitive ring. We claim $R / J$ has a faithful semi-simple left $R$-module. Since each $R_{\alpha}$ is primitive, it has a faithful simple left $R_{\alpha}$-module, say $S_{\alpha}$. Clearly each $S_{\alpha}$ is then a simple $R / J$ module, so

$$
T=\oplus \sum_{a \in A} S_{\alpha}
$$

is a semi-simple $R / J$ module. Since the $R / J$ annihilator of $T$ is just $\operatorname{ker} \rho=0, T$ is faithful.

Now let $S$ be any semi-simple left $R$-module. Then $S \oplus T$ is a faithful semisimple module over $R / J$, and is quasi-injective over $R / J$. So by [10, Theorem 1.2] and by hypothesis $S \oplus T$ is injective over $R / J$. Therefore $S$ is injective over $R / J$. This proves (a).

For (b) we observe that (a) says direct sums of injective hulls of simples are injective over $R / J$. Thus $R / J$ is left noetherian. [See 23]. This completes the proof.

\section{Quasi-projective modules}

Earlier we defined the notion of relative injectivity. We used relative injectivity to determine certain properties of quasi-injective modules. Here we shall consider the dual notion of relative projectivity, and use this concept to determine the structure of quasi-projective modules for certain types of rings.

THEOREM 2.1. Let $R$ be left perfect. Then the following are equivalent for $M$ a left $R$-module:

(a) $M$ is projective.

(b) $M$ is projective relative to $R$.

(c) $\operatorname{Ext}_{R}^{\prime}(M, I)=0$ for all $I \leqq{ }_{R} R$.

(d) For $K$ a superfiuous left ideal of $R$ and each $R$-homomorphism $\sigma: M \rightarrow R / K$, there exists $\sigma^{\prime}: M \rightarrow R$ such $\pi \sigma^{\prime}=\sigma$, where $\pi: R \rightarrow R / K$ is the natural epimorphism.

Proof. (a) $\Rightarrow$ (b): Clear.

(b) $\Rightarrow(a)$ : There exists a set $B$ and an epimorphism $\theta$ such that

$$
R^{(B)}=\oplus \sum_{B} R \stackrel{\theta}{\rightarrow} M \rightarrow 0 .
$$

This implies $M$ is projective relative to $R^{B}$ [see 27]. So since $R^{(B)} \leqq R^{B}$, by [27, Proposition 1], $M$ is projective relative to $R^{(B)}$. Thus, we have that $\theta$ splits and $M$ is projective. 
(c) $\Rightarrow$ (b): Assuming (c) we have the exactness of $\operatorname{Hom}_{R}(M, R) \rightarrow$ $\operatorname{Hom}_{R}(M, R / I) \rightarrow 0$.

Therefore, $M$ is clearly projective relative to $R$.

(d) $\Rightarrow$ (a): Consider

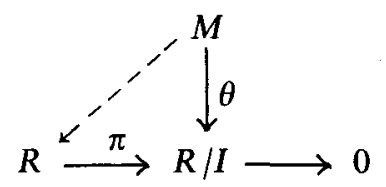

Since $R$ is left perfect, $R / I$ has a projective cover $P^{\prime}$; so by the projectivity of $R$, we have the commutative diagram:

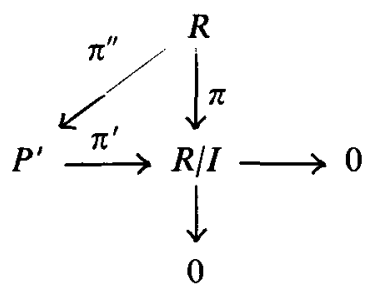

with $\operatorname{ker}\left(\pi^{\prime}\right)=K$ superfluous in $P^{\prime}$. Since $K$ is superfluous in $P^{\prime}$, one easily checks that $\pi^{\prime \prime}$ is onto. Thus since $P^{\prime}$ is projective, we may assume that $R=P^{\prime} \oplus P^{\prime \prime}$. Clearly then, $K$ is superfluous in $R$. Now observing that

$$
R / K=P^{\prime} \oplus P^{\prime \prime} / K \simeq P^{\prime} / K \oplus P^{\prime \prime} \simeq R / I \oplus P^{\prime \prime}
$$

we have

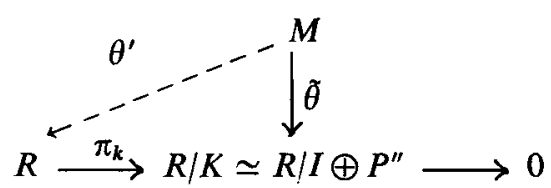

Here $\tilde{\theta}(M)=(\theta(M), 0)$. By hypothesis there exist $\theta^{\prime}: M \rightarrow R$ such that $\pi_{k} \theta^{\prime}=\tilde{\theta}$.

One now easily checks that $\pi \theta^{\prime}=\theta$.

(a) $\Rightarrow$ (c) and (a) $\Rightarrow$ (d) are trivial.

CoRollary 2.2. Let $R$ be a ring. Suppose $M$ has a projective cover $P(M)$, then $M$ is projective if and only if $M$ is projective relative to some faithful left $R$-module $N$.

Proof. It will suffice to prove the sufficiency of the condition. But if $N$ is faithful, there is a set $B$ such that $R \leqq N^{B}$ [26, Theorem 2]. So $M$ is projective relative to $N^{B}$. [See 27]. Hence if $M$ is projective relative to $N^{B}$, then $M$ is projective relative to $R$. So it follows easily from the proof of 2.1 that $M$ is projective. 
Remark 2.3. Theorem 2.1 does not hold in general. One can show that if $R=Z=$ integers, we have that $Q=$ rationals is projective relative to $Z$. But $Q$ is not projective.

An $R$-module $M$ is projective as an $R / l_{R}(M)$ if and only if $R$ is projective relative to $M^{\boldsymbol{A}}$ for every set $A$. (See [12].) We note that every (finitely generated) quasi-projective left $R$-module has this property if $R$ is left perfect (semi-perfect). However, in general this condition does not hold for an arbitrary quasi-projective module. (See [10].) We will give some necessary conditions on a ring $R$ in order that every quasi-projective be projective modulo its annihilator.

Recall that a ring is called semi-local in case $R / J$ is semi-simple where $J$ is the Jacobson radical.

${ }^{2}$ TheOREM 2.4. A ring $R$ is semi-local if and only if every semi-simple left $R$-module $M$ is projective over $R / l_{R}(M)$.

Proof. $(\Rightarrow)$ Each semi-simple left $R$-module $S$ is an $R / J$ module over a semi-local ring, and hence is $R / J$ projective. Noting that as $l_{R}(S) \geqq J$, every $R / l_{R}(S)$ module is an $R / J$-Module, one easily sees that $S$ is $R / l_{R}(S)$-projective.

$(\Leftrightarrow)$ Construct $T$ a faithful semi-simple $R / J$ module as in the proof of (1.5). Let $S$ be any simple $R / J$ module. Since $T \oplus S$ is faithful quasi-projective over $R / J$, by hypothesis $T \oplus S$ is projective over $R / J$. Therefore, every simple is projective over $R / J$, so $R / J$ is semi-simple.

We have necessary conditions on a ring $R$ for every quasi-projective left $R$-module to be projective modulo its annihilator.

It is not true that we may replace 'semi-simple' with 'simple' in (2.4) as the following proposition will show:

Proposition 2.5. Let $R$ be a commutative ring. Let $M$ be a finitely generated quasi-projective $R$-module, then $M$ is projective over $R / l_{R}(M)$.

Proof. Let $M$ be a finitely generated quasi-projective and let $m_{1}, \cdots, m_{k}$ be a set of generators for $M$. Define

$$
\phi: R \rightarrow \oplus \sum_{\alpha=1}^{k} M_{\alpha}, M_{\alpha} \cong M
$$

via $\pi_{\alpha} \phi(r)=r m_{\alpha}$. This is clearly an $R$-homomorphism. If $r \in \operatorname{ker} \phi$, then since $R$ is commutative,

$$
r\left(\sum_{\alpha=1}^{k} a_{\alpha} m_{\alpha}\right)=0
$$

whence $\operatorname{ker} \phi=l_{R}(M)$. So $\bar{R}=R / l_{R}(M)$ can be embedded in $\oplus \Sigma^{k} M_{x}$. So by

2 Kent Fuller has recently informed the author that he has recently proved a version of 2.4. See (II). 
[27, Proposition 1] $M$ is projective relative to $\bar{R}$. But, $M$ is finitely generated so we have

$$
\bar{R}^{(k)} \stackrel{\pi}{\rightarrow} M \rightarrow 0 .
$$

By (27, Proposition 1) again, $M$ is projective relative to $\bar{R}^{(k)}$, hence $\pi$ splits, so $M$ is $\bar{R}$ projective.

\section{Modules which are flat modulo their annihilator}

In the next section we introduce the concept of quasi-flat modules. These are designed to generalize flat modules in the same way that quasi-injectivity and quasi-projectivity generalized injectivity and projectivity.

If $M$ is a left $R$-module and $N$ is a right $R$-module, then $M$ is flat relative to $N$ in case for every monomorphism

the induced map

$$
f: K \rightarrow N
$$

$$
f \otimes 1: K \otimes_{R} M \rightarrow N \otimes{ }_{R} M
$$

is a monomorphism. Thus $M$ is flat if and only if it is flat relative to every right $R$-module.

Let $\mathscr{S}$ denote a subclass of right $R$-modules. Let $\mathscr{C}$ denote the smallest full abelian subcategory of $\mathscr{M}_{R}$ possessing the following properties:

(1) $\mathscr{S}$ is contained in $\mathscr{C}$.

(2) All modules of $\mathscr{M}_{R}$ isomorphic to a module of $\mathscr{C}$ is an object of $\mathscr{C}$.

(3) All submodules and factor modules of modules in $\mathscr{S}$ are in $\mathscr{C}$.

Robert [27] showed that $M$ is flat relative to $\mathscr{S}$ if and only if $M$ is flat relative to each module in $\mathscr{C}$, the category generated by $\mathscr{S}$.

There is a sort of functional dual between flat and injective modules that we wish to exploit. To do this consider the divisible abelian group $T=Q / Z$.

Note that this is actually an injective cogenerator for the category ${ }_{z} \mathscr{M}$ of abelian groups. For each left (right) $R$-module $M$ define its $T$-dual to be the right (left) $R$-module: $M^{*}=\operatorname{Hom}_{\mathrm{z}}(M, T)$ where $M^{*}$ is a right $R$-module via $(x, r)(m)$ $=x(r m)$ where $x \in M^{*}, r \in R, m \in M$. More generally, if $D$ is a divisible $Z$-module, and if $M$ is a left (right) $R$-module, The $D$-dual of $M$ is $M_{D}^{*}=\operatorname{Hom}_{\mathrm{Z}}(M, D)$.

THEOREM 3.1 (Lambek [24]). Let $R$ be a ring and $M$ a left $R$-module. Then the following are equivalent:

(a) $M$ is flat.

(b) $M^{*}$ is injective (as a right $R$-module).

(c) $M_{D}^{*}$ is injective (as a right $R$-module) for every divisible group $D$.

The effect of (3.1) is that we can analyze the flatness of ${ }_{R} M$ in terms of the injectivity of its "dual module" $M^{*}$. Now using well known properties of $M^{*}$, it is easy to prove the following. 
Lemma 3.2. Let $R$ be a ring, and let $M, N, K$ be left $R$-modules. Then $\mathscr{M}_{R}$.

(a) $K \stackrel{f}{\rightarrow} M \stackrel{g}{\rightarrow} N$ is exact in $R^{\mathscr{M}}$ if and only if $N^{*} \stackrel{g^{*}}{\rightarrow} M^{*} \stackrel{f^{*}}{\rightarrow} K^{*}$ is exact in

(b) $M$ is faithful as a left $R$-module if and only if $M^{*}$ is faithful as a right R-module.

(c) $l_{R}(M)=r_{R}\left(M^{*}\right)$.

Proof. (a) is [24, Proposition 1, p. 127].

For (b) assume $M$ is faithful. Let $0 \neq r \in R$. Then there exists $m \in M$ such that $r \cdot m \neq 0$. By [25, Lemma 1], there exists $x \in M^{*}$ such that $x(r m) \neq 0$; i.e. $x(r m)=(x \cdot r)(m) \neq 0$. So $M^{*}$ is faithful. That $M^{*}$ is faithful implies $M$ faithful is trivial. Finally, it is clear that (c) follows from (b).

Now suppose that $M$ is a left $R$-module. Then its annihilator $l_{R}(M)$ is an ideal of $R$ and $M$ is naturally a left module over the factor ring $R=R / l_{R}(M)$. By (3.2), its dual $M^{*}$ is also a right module over $\bar{R}$, and clearly, if $K$ is an $R$ (hence $\bar{R}$ ) submodule of $M^{*}$, then

$$
K \otimes_{R}(M)=K \otimes_{R_{R}} M .
$$

Now let $R$ be a ring and $M$ a left $R$-module. We say $M$ is quasi-flat in case for every divisible abelian group $D, M$ is flat relative to

$$
M_{D}^{*}=\operatorname{Hom}_{\mathrm{Z}}(M, D) .
$$

In other words, $M$ is quasi-flat if and only if for every such $D$ and every right $R$-submodule $K \leqq K_{D}^{*}$ the natural map $K \otimes_{R} M \rightarrow M_{D}^{*} \otimes{ }_{R} M$ is a monomorphism. Clearly, any flat module is quasi-flat, but not every quasi-flat module is flat as is shown in the following example.

EXAmPLE 3.3. Let $R=Z$ and consider the simple $Z$-module $Z_{p}$ for $p$ a prime. Then since

$$
Z / l_{Z}\left(Z_{p}\right)=Z /(p) \cong Z_{p}
$$

it is clear that $Z_{p}$ is actually flat as a $Z_{p}$-module; hence $Z_{p}$ is quasi-flat as a $Z$ module as we shall see from (3.4). But since $Z_{p}$ is not torsion free, it is not flat over $Z$.

Our main result characterising quasi-flat modules is the following.

THEOREM 3.4. Let $R$ be a ring, and let $M$ be a left $R-M o d u l e$, let $l_{R}(M)$ be its left annihilator and let

$$
\bar{R}=R / l_{R}(M)
$$

Then the following are equivalent: 
(a) $M$ is quasi-flat.

(b) $M$ is flat over $\tilde{R}$.

(c) $M$ is flat relative to $\left(M^{*}\right)^{A}$ for every set $A$.

(d) $M^{*}$ is $\Pi$-quasi-injective as a right $R$-module.

(e) $M_{D}^{*}$ is quasi-injective for every divisible ${ }_{2} D$.

(f) $M_{D}^{*}$ is $\Pi$-quasi-injective for every divisible ${ }_{z} D$.

Proof. (d) $\Leftrightarrow$ (e) $\Leftrightarrow$ (f): These implications all follow from the facts that every product of divisible groups is divisible, that every divisible group is a direct summand of some power $(Q / Z)^{A}$ of $Q / Z$, and that for every set $A$

$$
\operatorname{Hom}_{\mathrm{Z}}\left(M,(D)^{A}\right) \cong \Pi_{A} \operatorname{Hom}_{\mathrm{Z}}(M, D) \text {. }
$$

(a) $\Rightarrow$ (e): Let ${ }_{z} D$ be divisible and let

$$
0 \rightarrow K \stackrel{f}{\rightarrow} M_{D}^{*}
$$

be a right $R$-homomorphism. By hypothesis (a), we have

$$
0 \rightarrow K \otimes_{R} M \stackrel{f \otimes 1}{\rightarrow} M_{D}^{*} \otimes_{R} M
$$

is a $Z$-monomorphism. Since ${ }_{\mathrm{Z}} D$ is injective,

$$
\left(M_{D}^{*} \otimes_{R} M\right) \rightarrow\left(K \otimes_{R} M\right)^{*} \rightarrow 0
$$

is exact. But there are natural isomorphisms

and

$$
\left(K \otimes_{R} M\right)^{*} \cong \operatorname{Hom}_{R}\left(K, M_{D}^{*}\right)
$$

$$
\left(M_{D}^{*} \otimes M\right)_{D}^{*} \cong \operatorname{Hom}_{R}\left(M_{D}^{*}, M_{D}^{*}\right) .
$$

(See [24, Proposition 3, p. 123].) Thus

$$
\operatorname{Hom}_{R}\left(M_{D}^{*}, M_{D}^{*}\right) \rightarrow \operatorname{Hom}_{R}\left(K, M_{D}^{*}\right) \rightarrow 0
$$

is exact, so that $M_{D}^{*}$ is quasi-injective.

(d) $\Rightarrow$ (b): Since $M^{*}$ is $\Pi$-quasi-injective over $R$ (by hypothesis), it is easily seen that $M^{*}$ is $\Pi$-quasi-injective over $\bar{R}$ (see 3.2). But since $M^{*}$ is faithful over $\bar{R}$, there is an embedding

$$
0 \rightarrow R \rightarrow\left(M^{*}\right)^{A}
$$

of $\bar{R}$ into some power of $M^{*}$ (see [26]). Thus, since $\left(M^{*}\right)^{A}$ is quasi-injective, it is injective over $\bar{R}$; hence $M^{*}$ is injective over $\bar{R}$ and $M$ is $\bar{R}$ flat by (3.1).

(b) $\Rightarrow$ (c): This is trivial since both $M$ and each $\left(M^{*}\right)^{A}$ are $\bar{R}$-modules (see $3: 1)$. 
(c) $\Rightarrow$ (a): Let ${ }_{Z} D$ be any divisible group. Since $T=Q / Z$ is an injective cogenerator for $Z$, there is some set $A$ and some split monomorphism

$$
0 \rightarrow D \rightarrow T^{A} \text {. }
$$

Thus, there is a split monomorphism

$$
0 \rightarrow M_{D}^{*} \rightarrow\left(M^{*}\right)^{A} .
$$

So clearly, if $M$ is flat relative to $\left(M^{*}\right)^{A}$, it is flat relative to the direct summand $M_{D}^{*}$.

REMARK 3.5. It is natural to ask whether a module $M$ is quasi-flat whenever it is flat relative to $M^{*}$. Although we do not know we suspect this is the case. It follows from (3.4) that this is equivalent to asking whether every quasi-injective dual is $\Pi$-quasi-injective.

Corollary 3.6. Let $M$ be a faithful left R-module. Then $M$ is flat if and only if $M$ is quasi-flat.

Proof. The proof is clear by (3.4) since $l_{R}(M)=0$.

\section{Some characterizations of rings using quasi-flat modules}

In this Section we will use the concepts developed in $\$ 3$ to characterize certain classes of rings.

A ring is Von Neumann if and only if every left $R$-module is flat. More generally we have:

Proposition 4.1. Let $R$ be a ring, then $R$ is von Neumann if and only if each left (right) $R$-module is quasi-flat.

Proof. Let $M$ be a finitely generated left $R$-module. Thus $M \oplus R$ is quasiflat. But $M \oplus R$ is faithful, so $M \oplus R$ is flat by 3.4. Therefore, $M$ is flat, and so $R$ is Von Neumann. The converse is trivial.

THEOREM 4.2. For a ring $R$ the following are equivalent:

(a) $R$ is left perfect.

(b) For a left R-module $M, M$ is quasi-projective if and only if $M$ is quasi-flat.

Proof. (a) $\Rightarrow$ (b): Let $M \in_{R} M$ be quasi-projective. Then by [12, Theorem 2.3] $M$ is projective over $R / l_{R}(M)$ so in particular, $M$ is flat over $R / l_{R}(M)$. So by (3.4) $M$ is quasi-flat as a left $R$-module.

Conversely, suppose $M$ is quasi-flat. Then $M$ is flat as an $R / l_{R}(M)$ module. So since $R / l_{R}(M)$ is left perfect, $M$ is projective over $R / l_{R}(M)$. (See Govorov [14].) Hence one easily checks that $M$ is quasi-projective as a left $R$-module. 
(b) $\Rightarrow$ (a): Suppose $M$ is flat. Then for every set $A, R^{(A)} \oplus M$ is flat. Hence, by the hypothesis, each $R^{(A)} \oplus M$ is quasi-projective. Thus, by [12, Corollary 1.2], $M$ is projective relative to $R^{(A)}$. But there is a set $A$ and an epimorphism

$$
R^{(A)} \stackrel{\stackrel{9}{\rightarrow}}{\rightarrow} M \rightarrow 0 .
$$

Thus $\theta$ must split, so $M$ is projective. Therefore, by [14] $R$ is left perfect.

${ }^{3}$ COROLlaRY 4.3. If every flat left $R$-module is quasi-projective, then $R$ is left perfect.

Proof. By 4.2.

A finitely generated left $R$-module $M$ is finitely related in case any exact sequence

$$
0 \rightarrow K \rightarrow F \rightarrow M \rightarrow 0
$$

with $F$ finitely generated free has the property that $K$ is finitely generated. A ring $R$ is called right coherent if each of its finitely generated right ideals is finitely related. Chase [4] proved that a ring $R$ has the property that every flat left $R$ module is $\Pi-$ flat if and only if $R$ is right coherent. We prove the following result.

THEOREM 4.4. For a ring $R$ the following are equivalent:

(a) Every factor ring of $R$ is right coherent.

(b) Every quasi-flat left $R$-module is $\Pi$-quasi-flat.

(c) $R / I$ is $\Pi$-quasi-flat (as an R-module) for every two-sided ideal I of $R$.

Proof. (a) $\Rightarrow$ (b): Suppose that $M$ is quasi-flat as a left $R$-module. Then $M$ is faithful over $R / l_{R}(M)$. So by (3.4), by the hypothesis, and by [4, Theorem 2.1], $M$ is $\Pi$-flat over $R / l_{R}(M)$. Therefore by (3.4) again $M$ is $\Pi$-quasi-flat as a left $R$-module.

(b) $\Rightarrow$ (c): Clear:

(c) $\Rightarrow$ (a): $R / I$ is flat as a module over itself. Hence is $\Pi$-flat as a module over itself. Hence by [4, Theorem 2.1] $R / I$ is right coherent.

Remark 4.5. There exist right coherent rings whose factor rings are not necessarily right coherent. Consider the ring $R=F\left[x_{1}, x_{2}, \cdots\right]$ of all polynomials over a field $F$ in an infinite number of indeterminates. This ring is right coherent, so products of flats are flats. However, consider the ideal $I$ consisting of nonlinear terms. Then $R / I=R^{\prime}$ is not coherent. For consider the principal ideal $I^{\prime}$ of $R / I=R^{\prime}$ generated by $x_{1}+I$. Let $K$ be the ideal of $R^{\prime}$ generated by $\left\{x_{i}+I i=1,2, \cdots\right\}$. Then $R^{\prime} / K$ is finitely generated, but is not finitely related. Now $I^{\prime} \simeq R^{\prime} / K$ via $a_{1} x_{1}+I \rightarrow a_{1}+K$, so $R^{\prime}$ is not right coherent.

\footnotetext{
${ }^{3}$ The author has been informed that J. Golan has proved a version of 4.3. See [13].
} 


\section{References}

[1] F. W. Anderson, Structure of rings and algebras (Lecture Notes, University of Oregon, 1966-67).

[2] H. Bass, 'Finistric dimension and a homological generalization of semi-primary rings', Trans . Amer. Math. Soc. 95 (1960), 466-488.

[3] N. Bourbaki, Algebre Commutative, Chapters I-II (Act. Sc. Ind. 1290, Paris: Hermann, 1961).

[4] S. Chase, 'Direct products of modules', Trans. Amer. Math. Soc. 95 (1960), 457-473.

[5] C. W. Curtis and I. Reiner, Representation Theory of Finite Groups and Associative Algebras (New York: Interscience, 1962).

[6] B. Eckman and A. Schopf, 'Über injecktive Moduln', Arch. Math. 4 (1953), 75-78.

[7] C. Faith, 'Rings with ascending condition on annihilators', Nagoya Math. J. 27 (1966), 179-191.

[8] C. Faith and Y. Utumi, 'Quasi-injective modules and their endomorphism rings', Arch. Math. 15 (1964), 166-174.

[9] K. Faith and E. A. Walker, 'Direct-sum representations of injective modules', J. Algebra 5 (1967), 203-221.

[10] K. R. Fuller, 'On direct representations of quasi-injectives and quasi projectives', Arch . Math. 20 (1969), 495-502.

[11] K. R. Fuller, 'Relative projectivity and injectivity classes determined by Simple Modules', (To appear).

[12] K. R. Fuller and D. A. Hill, 'On quasi-projective modules via relative projectivity', (To appear).

[13] J. S. Golan, 'Characterizations of rings using quasi-projective modules', Israel J. Math. 8 (1970), 34-38.

[14] V. E. Govorov, 'Rings over which flat modules are free', Soviet Math. 3 (1962), 836-838.

[15] M. Harada, 'Note on quasi-injective modules', Osaka J. Math. 2 (1965), 351-356.

[16] N. Jacobson, Structure of Rings, Rev. Ed. (Providence, 1964).

[17] J. P. Jans, 'Projective injective modules', Pacific J. Math. 9 (1959), 1103-1108.

[18] J. P. Jans, Rings and Homology (New York: Holt, Rinehart, and Winston, 1964).

[19] J. Jans and L. E. T. Wu, 'On quasi-projectives', Illinois J. Math. 11 (1957), 439-448.

[20] R. E. Johnson and E. T. Wong, 'Quasi-injective modules and irreducible rings', J. London Math. Soc. 36 (1961), 260-268.

[21] A. Koehler, 'Quasi-projective and quasi-injective modules', (To appear).

[22] A. Koehler, 'Quasi-projective covers and direct sums', (To appear).

[23]. R. P. Kurshen, Injective modules with a finitely generated essential socle (Ph. D. thes is, Notices Amer. Math. Soc., 16 (1969).

[24] J. Lambek, Lectures on Rings and Modules (Waltham, Massachusetts: Blaisdell, 1966).

[25] J. Lambek, 'A module is flat if and only if its character module is injective', Canad. Math. Bull. 7 (1963), 237-243.

[26] K. Morita, 'On the S-rings in the sense of F. Kasch', Nagoya Math. J. 27 (1966), 687-695.

[27] E. Robert, 'Projectives et injectives relatifs', C. R. Acad. Sci. Paris Ser. A. B. 286 (1960), Ser. A. 361-364.

Department of Mathematics

University of Western Australia. 